\title{
Análise das ações dos estados e Distrito Federal para segurança alimentar e nutricional na pandemia de Covid-19 no Brasil
}

\author{
Analysis of states and Federal District actions for food and nutritional security in the Covid-19 \\ pandemic in Brazil \\ Análisis de las acciones de los estados y del Distrito Federal para la seguridad alimentaria y \\ nutricional en la pandemia de Covid-19 en Brasil
}

\section{Resumo}

O objetivo deste estudo foi analisar as propostas emitidas pelos governos estaduais e do Distrito Federal para o enfrentamento da insegurança alimentar e nutricional durante a pandemia de Covid-19 no Brasil. Através da análise documental foram verificados os atos oficiais publicados pelos estados e Distrito Federal para mitigar a insegurança alimentar e nutricional. Após a busca e leitura prévia dos documentos foram selecionados 55 atos, sendo 31 (56,4\%) decretos, $23(41,8 \%)$ leis e $1(1,8 \%)$ ordem de serviço. Constatou-se que $34(61,8 \%)$ documentos mencionavam a articulação com municípios para a realização de ações em segurança alimentar e nutricional e 37 (67,3\%) atos mencionavam ações intra ou intersetoriais. Prevaleceram abordagens envolvendo alimentação escolar, aspectos gerais em segurança alimentar e nutricional e distribuição de cestas básicas de alimentos. Foi verificada uma disparidade nas ações estaduais e distritais, não apenas em número, mas também na abrangência e especificidade das propostas. Desta forma, torna-se importante o fortalecimento institucional e integração dos estados e Distrito Federal na gestão das ações de segurança alimentar e nutricional para a garantia do direito humano à alimentação adequada.

Palavras-chave: COVID-19; Segurança alimentar; Política pública; Legislação; Programas de nutrição.

\begin{abstract}
The aim of this study was to analyze the proposals published by the state and Federal District governments to deal with food and nutritional insecurity during the COVID-19 pandemic in Brazil. By the documental analysis, were verified the official acts published by the states and the Federal District to mitigate food and nutritional insecurity. After searching and prior reading of the documents, a total of 55 acts, $31(56.4 \%)$ decrees, $23(41.8 \%)$ laws and $1(1.8 \%)$ service order. It was found that $34(61.8 \%)$ documents mentioned the articulation with municipalities to carry out actions in food and nutritional security and 37 (67.3\%) acts mentioned intra or intersectoral actions. Predominated approaches involving school feeding, general aspects in food and nutritional security and distribution of staple basket food. There was a disparity in state and district actions, not only in number, but also in the scope and specificity of the proposals. Thus, it is important to institutionally strengthen and integrate the states and the Federal District in the management of food and nutrition security actions to guarantee the human right to adequate food.
\end{abstract}

Keywords: COVID-19; Food security; Public policy; Legislation; Nutrition programs.

\section{Resumen}

El objetivo de este estudio fue analizar las propuestas emitidas por los gobiernos estatales y del Distrito Federal para abordar la inseguridad alimentaria y nutricional durante la pandemia de Covid-19 en Brasil. Mediante análisis documental, se verificaron los actos oficiales publicados por los estados y el Distrito Federal para mitigar la inseguridad 
alimentaria y nutricional. Después de la búsqueda y lectura previa de los documentos, se seleccionaron 55 actos, 31 $(56,4 \%)$ decretos, $23(41,8 \%)$ leyes y $1(1,8 \%)$ orden de servicio. Se encontró que $34(61,8 \%)$ documentos mencionaron la articulación con los municipios para llevar a cabo acciones en seguridad alimentaria y nutricional y 37 (67,3\%) actos mencionados como acciones intra o intersectoriales. Predominaron los enfoques que implicaban la alimentación escolar, los aspectos generales de la seguridad alimentaria y nutricional y la distribución de canastas básicas de alimentos. Hubo una disparidad en las acciones estatales y distritales, no sólo en número, sino también en el alcance y especificidad de las propuestas. Por ello, es importante fortalecer e integrar institucionalmente a los estados y al Distrito Federal en la gestión de las acciones de seguridad alimentaria y nutricional para garantizar el derecho humano a una alimentación adecuada.

Palabras clave: COVID-19; Seguridad alimentaria; Política pública; Legislación; Programas de nutrición.

\section{Introdução}

A pandemia de Covid-19 tornou evidente as desigualdades sociais marcadas pela ameaça ao direito humano à alimentação adequada (DHAA), em decorrência das restrições de renda que dificultam o acesso regular aos alimentos (Silva Filho \& Gomes Júnior, 2020). Estes eventos contribuem para instauração de situações de insegurança alimentar e nutricional (INSAN) no Brasil, com anuência do atual Governo Federal que suprime a agenda governamental voltada para o DHAA, sendo este sistema norteado por políticas econômicas neoliberais ineficientes para a garantia das necessidades básicas da população (Silva Filho \& Gomes Júnior, 2020).

Previa-se que a pandemia de Covid-19 poderia impactar negativamente na segurança alimentar e nutricional (SAN), com potenciais efeitos heterogêneos que influenciam nos diferentes fatores de risco atrelados ao estado nutricional, como obesidade e má nutrição, que podem ser agravados quando a resposta do poder público não ocorre com a devida urgência (Jaime, 2020).

O amplo conceito de SAN possibilita a compreensão de que a alimentação é um direito humano que se relaciona a outros direitos fundamentais tais como saúde, moradia, educação, trabalho, participação e informação (Guerra et al., 2019; Lei n. 11.346, 2006).

A INSAN é um desafio a ser superado para o pleno desenvolvimento dos países e da sociedade, de modo que esta questão integra a Agenda 2030 dos Objetivos do Desenvolvimento Sustentável (ODS) elaborada pela Organização das Nações Unidas (ONU) em 2015. A fome e a desnutrição, a dificuldade de acesso regular a alimentos seguros e nutritivos, e os entraves para o estabelecimento de sistemas biologicamente e economicamente sustentáveis compõem obstáculos contemporâneos para a SAN (Ministério da Cidadania, 2016; Ministério do Planejamento, Desenvolvimento e Gestão, 2018).

Este cenário preocupante em torno da INSAN se torna ainda mais alarmante frente a pandemia de Covid-19, em que a paralisação de algumas atividades econômicas impactou diretamente na renda de milhões de pessoas (Sipioni et al., 2020). Além disso, este evento afeta negativamente os sistemas alimentares ao distorcer a oferta e a demanda, degradar o poder de compra da população e reduzir a capacidade de produção e distribuição de alimentos (Erokhin \& Gao, 2020).

O enfrentamento desta emergência demanda que o Estado cumpra efetivamente o seu papel, com vistas a abrandar os efeitos da pandemia. As medidas de enfrentamento não devem ser direcionadas somente para o controle da disseminação do vírus, mas também para as repercussões que ocorrerão a curto, médio e longo prazo, no âmbito social e econômico (Barreto et al., 2020).

Desde o início da pandemia algumas medidas foram estabelecidas, em nível federal, inclusive no contexto das diretrizes definidas pela Política Nacional de Segurança Alimentar e Nutricional (PNSAN) (Alpino et al., 2020). Instituída em 2010, essa política pública busca estruturar o Sistema Nacional de Segurança Alimentar e Nutricional (SISAN) em um modelo que deve ser replicado nas três esferas de governo, por meio da adesão dos níveis estadual e municipal, através de ações pautadas em construções participativas e intersetoriais (Machado et al., 2018). Ainda que identificadas, as medidas estabelecidas são 
entendidas como normativas com possíveis descompassos quanto às suas implementações nos contextos locais. Além disso, alguns entes federados sequer estão implementando estratégias para a garantia do direito à alimentação (Alpino et al., 2020).

Frente ao exposto, torna-se fundamental investigar se as ações realizadas no âmbito estadual e distrital estão em consonância com políticas públicas e equipamentos públicos mobilizados para SAN, como meios de se garantir o DHAA durante este período de pandemia (Jaime, 2020). Nesse sentido, o objetivo deste estudo foi analisar os atos normativos emitidos no âmbito estadual e distrital com propostas para o enfrentamento da insegurança alimentar e nutricional durante a pandemia de Covid-19 no Brasil.

\section{Metodologia}

Foi realizado um estudo exploratório-descritivo, de natureza aplicada com abordagem qualitativa, mediante análise documental para avaliar as ações em SAN, realizadas pelos governos estaduais e do Distrito Federal, durante o período de emergência em decorrência da Covid-19 no Brasil. A análise documental é um procedimento sistemático destinado à revisão ou avaliação de materiais impressos e eletrônicos que se concentra na produção ou reelaboração de conhecimentos (Bowen, 2009), sendo o delineamento mais antigo para a realização de pesquisas (Günther, 2006). Tal metodologia de pesquisa qualitativa pode ser entendida como a investigação baseada na extração e criação de informações para a compreensão de um fenômeno (Bowen, 2009; Kripka et al., 2015a). Para o desenvolvimento da pesquisa qualitativa com abordagem em análise documental são requeridas sistematizações para escolhas do tipo de documento, tipo de acesso e estruturação da análise (Godoy, 1995; Kripka et al., 2015a). Portanto, a análise documental tem como objetivo a formatação conveniente de informações por meio de transformações, buscando facilitar o acesso ao observador para consulta e referenciação (Bardin, 1977). A base da análise documental envolve a classificação-indexação com representação condensada da informação (Bardin, 1977), a qual realiza a caracterização do documento, a codificação, os registros, categorização, análise crítica e interpretativa (Godoy, 1995; Kripka et al., 2015b).

A realização deste estudo a partir da análise documental, insere-se na proposta de pesquisa qualitativa aplicada em diversos estudos da área de alimentação e nutrição, capaz de abordar temáticas sobre dimensões sociais, culturais, cognitivas, psicológicas, educacionais, políticas e comportamentais sobre alimentação e nutrição (Canesqui, 2009).

A escolha dos atos oficiais no âmbito das esferas dos governos estaduais e distrital deve-se ao processo de enfraquecimento das ações em SAN no âmbito federal, em decorrência da extinção do Conselho Nacional de Segurança Alimentar e Nutricional (CONSEA) no início de 2019, somado a adoção de políticas econômicas de austeridade fiscal que afetaram o SISAN (Ribeiro-Silva et al., 2020). Desta forma, este trabalho buscou verificar quais as iniciativas propostas pelos estados e Distrito Federal para mitigar a INSAN durante a pandemia, no contexto do esvaziamento da agenda do governo federal para a resolução de tais questões.

A pesquisa dos atos oficiais publicados pelos governos dos estados e do Distrito Federal foi realizada no site "Leis Estaduais", disponível no endereço https://leisestaduais.com.br/. Foram selecionados os atos publicados pelas unidades federativas entre 6 de fevereiro de 2020 a 14 de agosto de 2020. Para a busca dos atos oficiais foram utilizados os seguintes termos e suas combinações: "alimentação" e "COVID-19", "alimentação" e "COVID", "alimentação" e "coronavírus", "alimentos" e "COVID-19", "alimentos" e "COVID", "alimentos" e "coronavírus", "segurança alimentar e nutricional" e "COVID-19", "segurança alimentar e nutricional" e "COVID", "segurança alimentar e nutricional" e "coronavírus", "segurança alimentar" e "COVID-19", "segurança alimentar" e "COVID”, "segurança alimentar" e "coronavírus".

Os documentos obtidos na etapa de busca à base de dados de legislações estaduais foram submetidos à leitura prévia. Em seguida, foram selecionados para a etapa de análise documental todos os atos que abordavam a temática de SAN, de acordo com a PNSAN e o Programa Nacional de Alimentação Escolar (PNAE), definindo os seguintes tipos de ações: (i) produção de 
alimentos; (ii) disponibilidade de alimentos; (iii) renda e condições de vida; (iv) acesso à alimentação adequada e saudável, incluindo água; (v) saúde, nutrição e acesso a serviços relacionados; (vi) alimentação escolar.

Os atos oficiais selecionados foram analisados a partir do preenchimento dos seguintes itens e critérios: (i) Sigla da unidade da federação responsável pela publicação do ato; (ii) Data de publicação; (iii) Tipo de ato normativo; (iv) Síntese da ação em SAN descrita; (v) Menção do termo "segurança alimentar e nutricional"; (vi) Verificação de propostas para articulação dos governos estaduais ou do Distrito Federal com os municípios para realização das ações em SAN; (vii) Especificação do ato oficial publicado; (viii) Menção de ações intra ou intersetoriais para SAN; ix) Análises estratificadas por unidades federativas.

\section{Resultados}

A busca pelos atos oficiais utilizando os descritores previamente determinados resultou na obtenção de 148 documentos. Após a etapa de leitura preliminar destes documentos, foram excluídos 93 atos por não cumprirem os critérios de elegibilidade, devido à ausência de descrição detalhada sobre ações voltadas para SAN. Ao final, foram selecionados 55 atos para análise documental por abrangerem a temática de SAN conforme as normativas da PNSAN e PNAE (Tabela 1).

Tabela 1. Análise descritiva dos atos normativos emitidos pelos governos estaduais e do Distrito Federal. Brasil, 2020.

\begin{tabular}{lr}
\hline Atributos avaliados & Total \\
\hline Atos encontrados & 148 \\
Atos descartados para análise documental & 93 \\
Atos selecionados para análise documental & 55
\end{tabular}

\section{Número de atos classificados de acordo com o tipo de ação em SAN}

Produção de alimentos

Disponibilidade de alimentos $\quad 55$

Renda e condições de vida $\quad 14$

Acesso à alimentação adequada e saudável, incluindo água 30

Saúde, nutrição e acesso a serviços relacionados $\quad 20$

Alimentação escolar 16

Tipo de ato selecionado para análise documental

Decreto

Lei 23

Ordem de serviço 1

Menciona o termo "segurança alimentar e nutricional"

Sim

$\begin{array}{lr}\text { Parcialmente } & 20\end{array}$

Não $\quad 19$

Menciona a articulação com municípios

Sim

Não $\quad 21$

Menciona alguma ação específica em SAN

Sim $\quad 30$

Não 25

Menciona alguma articulação intra ou intersetorial

Sim

Não 18 
No período analisado foram emitidos atos na forma de decretos $(56,4 \%)$, leis $(41,8 \%)$ e ordem de serviço $(1,8 \%)$, conforme observado na Tabela 1. Verificou-se que 34,5\% dos atos não descreveram integralmente ou parcialmente o termo "segurança alimentar e nutricional". O acesso à alimentação adequada e saudável, incluindo água, foi descrito em 54,4\% dos atos. Ações referentes à saúde, nutrição e acesso a serviços relacionados estavam presentes em $34,4 \%$ dos atos. A maior parte dos documentos $(61,8 \%)$ mencionou intenções quanto a articulações com municípios para a realização das ações. A articulação de ações intra ou intersetoriais foi citada na maioria dos documentos analisados $(67,3 \%)$.

Observa-se na Tabela 2, que a região Sudeste foi a que mais emitiu atos no período analisado (32,7\%), seguido pelas regiões Nordeste $(25,5 \%)$, Norte $(20 \%)$ e Centro-Oeste $(14,5 \%)$. Nota-se ainda que a região Sul foi a localidade com a menor publicação $(7,3 \%)$.

Tabela 2. Análise descritiva dos atos oficiais publicados pelos governos dos estados e Distrito Federal por regiões. Brasil, 2020.

\begin{tabular}{|c|c|c|c|c|c|c|c|c|c|c|c|c|}
\hline Especificação da ação atribuída ao ato & $\mathbf{N}$ & NE & & $\begin{array}{l}\text { SE } \\
\text { ita }(\end{array}$ & & Total & $\mathbf{N}$ & $\mathbf{N E}$ & $\begin{array}{l}\text { CO } \\
\text { Relati }\end{array}$ & $\begin{array}{c}\mathrm{SE} \\
\mathrm{va}(\%)\end{array}$ & $\mathbf{S}$ & Total \\
\hline Alimentação escolar & 1 & 4 & 3 & 5 & 1 & 14 & 9,1 & 28,6 & 37,5 & 27,8 & 25,0 & 25,5 \\
\hline Segurança alimentar e nutricional & 2 & 4 & 2 & 2 & 2 & 12 & 18,2 & 28,6 & 25,0 & 11,1 & 50,0 & 21,8 \\
\hline Distribuição de cestas básicas & 0 & 3 & 1 & 2 & 0 & 6 & 0,0 & 21,4 & 12,5 & 11,1 & 0,0 & 10,9 \\
\hline $\begin{array}{l}\text { Auxílio destinado exclusivamente para } \\
\text { aquisiçãao de gênero alimentício }\end{array}$ & 0 & 0 & 0 & 0 & 1 & 1 & 0,0 & 0,0 & 0,0 & 0,0 & 25,0 & 1,8 \\
\hline $\begin{array}{l}\text { Aborda o tema sobre o recebimento de } \\
\text { doações }\end{array}$ & 0 & 0 & 2 & 1 & 0 & 3 & 0,0 & 0,0 & 25,0 & 5,6 & 0,0 & 5,5 \\
\hline $\begin{array}{l}\text { Auxílio financeiro às famílias em } \\
\text { situação de vulnerabilidade social }\end{array}$ & 2 & 0 & 0 & 1 & 0 & 3 & 18,2 & 0,0 & 0,0 & 5,6 & 0,0 & 5,5 \\
\hline $\begin{array}{l}\text { Estabelecimento de limite de produtos } \\
\text { adquiridos por cliente no comércio } \\
\text { (alimentação) }\end{array}$ & 6 & 2 & 0 & 0 & 0 & 8 & 54,5 & 14,3 & 0,0 & 0,0 & 0,0 & 14,5 \\
\hline $\begin{array}{l}\text { Acolhimento e proteção de mulheres } \\
\text { vítimas de violência doméstica e familiar } \\
\text { e seus dependentes, incluindo serviço de } \\
\text { alimentação }\end{array}$ & 0 & 0 & 0 & 1 & 0 & 1 & 0,0 & 0,0 & 0,0 & 5,6 & 0,0 & 1,8 \\
\hline $\begin{array}{l}\text { Relata sobre a manutenção do auxílio- } \\
\text { refeição ou alimentação para os } \\
\text { servidores estaduais }\end{array}$ & 0 & 0 & 0 & 1 & 0 & 1 & 0,0 & 0,0 & 0,0 & 5,6 & 0,0 & 1,8 \\
\hline $\begin{array}{l}\text { Fornecimento de alimentos às famílias } \\
\text { em situação de vulnerabilidade social }\end{array}$ & 0 & 0 & 0 & 3 & 0 & 3 & 0,0 & 0,0 & 0,0 & 16,7 & 0,0 & 5,5 \\
\hline $\begin{array}{l}\text { Distribuição de refeições para populações } \\
\text { carentes e pessoas em situação de rua }\end{array}$ & 0 & 0 & 0 & 2 & 0 & 2 & 0,0 & 0,0 & 0,0 & 11,1 & 0,0 & 3,6 \\
\hline $\begin{array}{l}\text { Fornecimento de alimentos aos } \\
\text { funcionários que trabalharem em prol ao } \\
\text { enfrentamento do SARS-CoV-2 }\end{array}$ & 0 & 1 & 0 & 0 & 0 & 1 & 0,0 & 7,1 & 0,0 & 0,0 & 0,0 & 1,8 \\
\hline Total & 11 & 14 & 8 & 18 & 4 & 55 & 100,0 & 100,0 & 100,0 & 100,0 & 100,0 & 100,0 \\
\hline
\end{tabular}

Legenda: (N) região Norte; (NE) região Nordeste; $(\mathrm{CO})$ região Centro-Oeste; (SE) região Sudeste; (S) região Sul; (n) número de atos oficiais; (\%) percentual relativo referente ao número de atos oficiais. Fonte: Autores (2021).

$\mathrm{Na}$ análise estratificada por unidades federativas, o estado do Rio de Janeiro foi responsável pela publicação do maior número de leis referentes às ações de SAN, seguido pelos estados de Minas Gerais, Ceará e São Paulo (Quadro 1). Em relação a publicação de decretos, as unidades federativas que mais emitiram tais atos foram Rondônia, São Paulo, Distrito Federal e Rio Grande do Norte. 
Quadro 1. Listagem e análise descritiva dos atos normativos para ações em SAN por regiões e unidades federativas. Brasil, 2020.

\begin{tabular}{|c|c|c|c|}
\hline UF & $\begin{array}{c}\text { Data de } \\
\text { publicação }\end{array}$ & Ato & Síntese da deliberação que contempla diretrizes, medidas e/ou ações em SAN \\
\hline \multicolumn{4}{|r|}{ Região Nordeste } \\
\hline AL & 07/05/2020 & Lei $\mathrm{n}^{\circ} 8.260$ & $\begin{array}{l}\text { Fornece alimentação de qualidade aos alunos da rede pública estadual de ensino durante } \\
\text { o período de férias, recesso escolar e suspensão das atividades. }\end{array}$ \\
\hline BA & 09/04/2020 & $\begin{array}{l}\text { Decreto }^{\circ} \\
19.627\end{array}$ & $\begin{array}{l}\text { Fornece alimentação aos voluntários que atuarem no enfrentamento do novo coronavírus } \\
\text { da Covid- } 19 \text {. }\end{array}$ \\
\hline BA & $14 / 04 / 2020$ & $\begin{array}{l}\text { Lei } n^{\circ} \\
14.259\end{array}$ & $\begin{array}{l}\text { Cria um projeto "Vale Alimentação Estudantil - PVAE", destinado à ações de } \\
\text { transferência de renda aos estudantes da rede pública estadual de ensino. }\end{array}$ \\
\hline $\mathrm{CE}$ & 09/04/2020 & $\begin{array}{c}\text { Decreto } \mathrm{n}^{\circ} \\
33.541\end{array}$ & $\begin{array}{l}\text { Institui o programa especial de alimentação estudantil voltado ao fornecimento de } \\
\text { alimentação aos alunos da rede Estadual de Ensino, durante a suspensão das atividades } \\
\text { presenciais. }\end{array}$ \\
\hline $\mathrm{CE}$ & $17 / 04 / 2020$ & $\begin{array}{l}\text { Lei } n^{\circ} \\
17.205\end{array}$ & $\begin{array}{l}\text { O Poder Executivo disponibiliza um auxílio financeiro às famílias de alunos da rede } \\
\text { pública estadual de ensino, para aquisição de produtos alimentícios, garantindo condições } \\
\text { mínimas de alimentação. }\end{array}$ \\
\hline $\mathrm{CE}$ & $27 / 03 / 2020$ & $\begin{array}{c}\text { Lei } \mathrm{n}^{\circ} \\
17.194\end{array}$ & $\begin{array}{l}\text { Estados e municípios realizam uma compra emergencial de cestas básicas para } \\
\text { fornecimento às famílias em situação de vulnerabilidade social, de modo a suprir as } \\
\text { necessidades alimentares. }\end{array}$ \\
\hline PB & 03/04/2020 & $\begin{array}{c}\text { Decreto } \mathrm{n}^{\circ} \\
40.167\end{array}$ & $\begin{array}{l}\text { Haverá um aumento de } \mathrm{R} \$ 15,00 \text { no Programa Cartão Alimentação e mais uma aquisição } \\
\text { emergencial de } 52 \text { mil cestas básicas e } 5 \text { mil kits de higiene para distribuição a pessoas } \\
\text { carentes. }\end{array}$ \\
\hline PB & $18 / 05 / 2020$ & $\begin{array}{l}\text { Decreto }^{\circ} \\
40.257\end{array}$ & $\begin{array}{l}\text { Determina a aquisição de } 60 \text { mil cestas básicas para distribuição com pessoas em condição } \\
\text { de vulnerabilidade social e um aporte de } \mathrm{R} \$ 1.000 .000,00 \text { para aquisição de gêneros } \\
\text { alimentícios para abastecimento e distribuição, preferencialmente, dos produtores da } \\
\text { Agricultura Familiar. }\end{array}$ \\
\hline PB & $10 / 06 / 2020$ & $\begin{array}{l}\text { Lei } n^{\circ} \\
11.703\end{array}$ & $\begin{array}{l}\text { Implementação de ações de SAN suficientes para proporcionar à população em situação } \\
\text { de rua acesso à alimentação de qualidade. }\end{array}$ \\
\hline $\mathrm{PE}$ & $22 / 03 / 2020$ & $\begin{array}{l}\text { Decreto }^{\circ} \\
48.835\end{array}$ & $\begin{array}{l}\text { Define medidas temporárias, que envolvem o abastecimento de água e segurança } \\
\text { alimentar. }\end{array}$ \\
\hline $\mathrm{RN}$ & $01 / 04 / 2020$ & $\begin{array}{c}\text { Decreto }^{\circ} \\
29.583\end{array}$ & $\begin{array}{l}\text { Fornecedores e comerciantes estabelecem limites de produtos por clientes para a } \\
\text { aquisição de bens essenciais como alimentação, a fim de que se evite escassez. }\end{array}$ \\
\hline $\mathrm{RN}$ & $14 / 04 / 2020$ & $\begin{array}{c}\text { Decreto }^{\circ} \\
29.613\end{array}$ & Criação do Programa Estadual Emergencial de Segurança Alimentar (RN Mais Unido). \\
\hline $\mathrm{RN}$ & $04 / 08 / 2020$ & $\begin{array}{l}\text { Decreto }^{\circ} \\
29.889\end{array}$ & $\begin{array}{l}\text { Criação do Programa Estadual Emergencial de Assistência Social (RN Chega Junto), de } \\
\text { caráter complementar e acessório à garantia constitucional ao direito à alimentação, à } \\
\text { integridade pessoal, à saúde, à assistência social e à vida. }\end{array}$ \\
\hline SE & $24 / 03 / 2020$ & $\begin{array}{l}\text { Decreto }^{\circ} \\
40.567\end{array}$ & $\begin{array}{l}\text { Fornecedores e comerciantes estabelecem limites de produtos por cliente para a aquisição } \\
\text { de bens essenciais como alimentação, a fim de que se evite escassez. }\end{array}$ \\
\hline \multicolumn{4}{|r|}{ Região Norte } \\
\hline $\mathrm{AM}$ & $07 / 04 / 2020$ & $\begin{array}{l}\text { Decreto }^{\circ} \\
42.166\end{array}$ & $\begin{array}{l}\text { Aquisição de produtos do setor primário para doação à população, como forma de } \\
\text { manutenção de segurança alimentar e garantia de renda mínima aos produtores rurais. }\end{array}$ \\
\hline AM & $01 / 06 / 2020$ & $\begin{array}{c}\text { Decreto }^{\circ} \\
42.354\end{array}$ & $\begin{array}{l}\text { Concessão de benefício eventual, para aquisição de gêneros alimentícios, de higiene e } \\
\text { limpeza de caráter provisório, às famílias em situação de extrema vulnerabilidade social. }\end{array}$ \\
\hline
\end{tabular}




\begin{tabular}{|c|c|c|c|}
\hline AM & $02 / 04 / 2020$ & Lei $\mathrm{n}^{\circ} 5.161$ & $\begin{array}{l}\text { Aquisição de insumos produzidos pelos produtores cadastrados na Agência de } \\
\text { Desenvolvimento Sustentável, a serem doados para as Instituições SEJUSC, SEAS e FPS, } \\
\text { atendendo a população suscetível aos riscos ocasionados pela falta de segurança } \\
\text { alimentar, bem como garantir a alimentação no período da pandemia. }\end{array}$ \\
\hline AP & $01 / 04 / 2020$ & Lei $\mathrm{n}^{\circ} 2.499$ & $\begin{array}{l}\text { Determina um auxílio financeiro emergencial para atender famílias em vulnerabilidade } \\
\text { social. }\end{array}$ \\
\hline RO & $20 / 03 / 2020$ & \begin{tabular}{c|} 
Decreto $\mathrm{n}^{\circ}$ \\
24.887
\end{tabular} & $\begin{array}{l}\text { Fornecedores e comerciantes estabelecem limites de produtos por cliente para a aquisição } \\
\text { de bens essenciais como alimentação, a fim de que se evite escassez. }\end{array}$ \\
\hline RO & $26 / 04 / 2020$ & \begin{tabular}{c|} 
Decreto $\mathrm{n}^{\circ}$ \\
24.979 \\
\end{tabular} & $\begin{array}{l}\text { Fornecedores e comerciantes estabelecem limites de produtos por cliente para a aquisição } \\
\text { de bens essenciais como alimentação, a fim de que se evite escassez. }\end{array}$ \\
\hline RO & $06 / 05 / 2020$ & \begin{tabular}{c|} 
Decreto $^{\circ}$ \\
25.008
\end{tabular} & $\begin{array}{l}\text { Transferência de recursos financeiros por meio de um cartão-alimentação aos genitores } \\
\text { ou responsáveis legais dos estudantes em situação de vulnerabilidade social matriculados } \\
\text { na rede pública de ensino, para aquisição direta de gêneros alimentícios. }\end{array}$ \\
\hline RO & $14 / 05 / 2020$ & $\begin{array}{c}\text { Decreto }^{\circ} \\
25.049\end{array}$ & $\begin{array}{l}\text { Fornecedores e comerciantes estabelecem limites de produtos por cliente para a aquisição } \\
\text { de bens essenciais como alimentação, a fim de que se evite escassez. }\end{array}$ \\
\hline $\mathrm{RO}$ & $10 / 06 / 2020$ & $\begin{array}{c}\text { Decreto }^{\circ} \\
25.130\end{array}$ & $\begin{array}{l}\text { Fornecedores e comerciantes estabelecem limites de produtos por cliente para a aquisição } \\
\text { de bens essenciais como alimentação, a fim de que se evite escassez. }\end{array}$ \\
\hline $\mathrm{RR}$ & $22 / 03 / 2020$ & \begin{tabular}{c|} 
Decreto $\mathrm{n}^{\circ}$ \\
28.635
\end{tabular} & $\begin{array}{l}\text { Fornecedores e comerciantes estabelecem limites de produtos por cliente para a aquisição } \\
\text { de bens essenciais como alimentação, a fim de que se evite escassez. }\end{array}$ \\
\hline TO & $21 / 03 / 2020$ & $\begin{array}{c}\text { Decreto } \mathrm{n}^{\circ} \\
6.072\end{array}$ & $\begin{array}{l}\text { Fornecedores e comerciantes estabelecem limites de produtos por cliente para a aquisição } \\
\text { de bens essenciais como alimentação, a fim de que se evite escassez. }\end{array}$ \\
\hline \multicolumn{4}{|r|}{ Região Centro-Oeste } \\
\hline $\mathrm{DF}$ & $23 / 03 / 2020$ & $\begin{array}{c}\text { Decreto }^{\circ} \\
40.550\end{array}$ & $\begin{array}{l}\text { As empresas que executarem tarefas vinculadas a assistência social, e nutrição, tanto para } \\
\text { o fornecimento de alimentação quanto para recolhimento e distribuição de alimentos em } \\
\text { programas para garantir a segurança alimentar, ficam excluídas da suspensão ocasionada } \\
\text { pela pandemia de Covid-19. }\end{array}$ \\
\hline DF & $01 / 04 / 2020$ & \begin{tabular}{c|} 
Decreto $^{\circ}$ \\
40.583
\end{tabular} & $\begin{array}{l}\text { Os alimentos destinados à merenda escolar, cuja data de validade esteja próxima do } \\
\text { vencimento, durante o período de suspensão das aulas da rede pública de ensino, deverão } \\
\text { ser destinados à Secretaria de Estado de Desenvolvimento Social. }\end{array}$ \\
\hline $\mathrm{DF}$ & 07/04/2020 & $\begin{array}{c}\text { Decreto }^{\circ} \\
40.604\end{array}$ & $\begin{array}{l}\text { O Instituto Banco de Brasília receberá doações de recursos financeiros para aquisição e } \\
\text { contratação, de forma imediata, de serviços, equipamentos, insumos, alimentos e demais } \\
\text { bens. }\end{array}$ \\
\hline DF & 09/04/2020 & $\begin{array}{c}\text { Decreto }^{\circ} \\
40.611\end{array}$ & $\begin{array}{l}\text { O programa "Todos Contra o COVID" poderá arrecadar, a título de doação, dinheiro e } \\
\text { outros recursos financeiros, por intermédio do Instituto BRB para aquisição e contratação, } \\
\text { de forma imediata, de serviços, equipamentos, insumos, alimentos e demais bens. }\end{array}$ \\
\hline $\mathrm{DF}$ & $25 / 05 / 2020$ & Lei $^{\circ} 6.588$ & $\begin{array}{l}\text { O Poder Executivo, através da Secretaria de Estado de Desenvolvimento Social, } \\
\text { disponibilizará cesta básica emergencial à população em situação de vulnerabilidade } \\
\text { social e insegurança alimentar. }\end{array}$ \\
\hline GO & $25 / 03 / 2020$ & \begin{tabular}{c|} 
Decreto $^{\circ}$ \\
9.643
\end{tabular} & $\begin{array}{l}\text { Alunos da rede pública de educação, cadastrados e beneficiados pelo programa Bolsa } \\
\text { Família ou equivalente, continuarão tendo direito à alimentação escolar durante o período } \\
\text { de suspensão das aulas. }\end{array}$ \\
\hline GO & 08/05/2020 & $\begin{array}{l}\text { Lei }^{\circ} \\
20.773\end{array}$ & $\begin{array}{l}\text { Será estabelecida condicionante na licença para empreendimentos voltada ao apoio e } \\
\text { fomento para atividades produtivas, promoção da SAN, geração de trabalho e renda. }\end{array}$ \\
\hline
\end{tabular}




\begin{tabular}{|c|c|c|c|}
\hline MT & $23 / 06 / 2020$ & $\begin{array}{l}\text { Lei } n^{\circ} \\
11.156\end{array}$ & $\begin{array}{l}\text { O Estado, em articulação com a União e com os Municípios, adotará medidas como } \\
\text { assistência alimentar às famílias de estudantes matriculados na educação básica da rede } \\
\text { estadual de ensino ou em instituição educacional conveniada com o Estado e proteção à } \\
\text { população em situação de rua, de modo a garantir a segurança alimentar e acesso à água } \\
\text { potável. }\end{array}$ \\
\hline \multicolumn{4}{|r|}{ Região Sul } \\
\hline $\mathrm{PR}$ & $04 / 05 / 2020$ & $\begin{array}{l}\text { Decreto }^{\circ} \\
4.570\end{array}$ & $\begin{array}{l}\text { Concessão de auxílio emergencial, destinado exclusivamente à aquisição de gêneros } \\
\text { alimentícios que compõem a cesta básica. }\end{array}$ \\
\hline RS & $20 / 04 / 2020$ & $\begin{array}{l}\text { Decreto }^{\circ} \\
55.197\end{array}$ & $\begin{array}{l}\text { Abertura de créditos extraordinários de } \mathrm{R} \$ 38.575 .000,00 \text {, sendo uma parte desse } \\
\text { montante destinado a gestão de política de SAN sustentável. }\end{array}$ \\
\hline $\mathrm{RS}$ & $17 / 07 / 2020$ & $\begin{array}{l}\text { Lei } n^{\circ} \\
15.488\end{array}$ & $\begin{array}{l}\text { Determina estratégias como: Apoio à alimentação escolar e Acesso à alimentação } \\
\text { adequada e saudável. }\end{array}$ \\
\hline $\mathrm{RS}$ & $24 / 07 / 2020$ & \begin{tabular}{|c|} 
Ordem de \\
Serviço do \\
Governador \\
$\mathrm{n}^{\circ} 009 / 2020$ \\
\end{tabular} & $\begin{array}{l}\text { O Comitê de Políticas Sociais e Educação promoverá ações voltadas à assistência social, } \\
\text { segurança alimentar e educação, discutidas e articuladas com os Secretários de Estado. }\end{array}$ \\
\hline \multicolumn{4}{|r|}{ Região Sudeste } \\
\hline MG & $02 / 04 / 2020$ & $\begin{array}{l}\text { Lei } n^{\circ} \\
23.631\end{array}$ & $\begin{array}{l}\text { Determina a concessão de benefícios como renda mínima emergencial e temporária para } \\
\text { segurança alimentar de grupos com vulnerabilidade socioeconômica, assistência } \\
\text { alimentar às famílias de estudantes do ensino público estadual e acesso à água potável. } \\
\text { Também foi determinado o estímulo à produção e à comercialização de alimentos da } \\
\text { agricultura familiar e de pequenos produtores, além de estimular a aquisição direta da } \\
\text { agricultura familiar no âmbito do PNAE. }\end{array}$ \\
\hline MG & $30 / 03 / 2020$ & $\begin{array}{c}\text { Decreto }^{\circ} \\
47.886\end{array}$ & Manutenção do auxílio-refeição ou alimentação para os servidores estaduais. \\
\hline MG & $10 / 04 / 2020$ & $\begin{array}{l}\text { Decreto } n^{\circ} \\
47.915\end{array}$ & $\begin{array}{l}\text { A Subsecretaria de Assistência Social da Secretaria de Estado de Desenvolvimento Social } \\
\text { realizará repasse financeiro ou fornecerá o cartão alimentação às famílias beneficiárias. }\end{array}$ \\
\hline MG & $04 / 06 / 2020$ & $\begin{array}{l}\text { Lei } n^{\circ} \\
23.652\end{array}$ & $\begin{array}{l}\text { Garantia da SAN para crianças e adolescentes, incluindo aqueles que vivem em } \\
\text { comunidades tradicionais. Será realizado um repasse de recursos do Fundo para Infância } \\
\text { e a Adolescência (FIA) para a garantia da proteção de crianças e adolescentes, incluindo } \\
\text { a SAN. }\end{array}$ \\
\hline MG & $15 / 06 / 2020$ & $\begin{array}{l}\text { Lei } n^{\circ} \\
23.660 \\
\end{array}$ & Traz a diretriz para garantia da segurança alimentar do idoso. \\
\hline $\mathrm{RJ}$ & $23 / 03 / 2020$ & Lei $\mathrm{n}^{\circ} 8.768$ & $\begin{array}{l}\text { Autoriza o Poder Executivo a realizar distribuição de cestas básicas para famílias de } \\
\text { estudantes da rede pública de ensino a partir do estoque de alimentos das escolas. }\end{array}$ \\
\hline RJ & $14 / 03 / 2020$ & Lei $\mathrm{n}^{\circ} 8.823$ & $\begin{array}{l}\text { As pessoas em situação de vulnerabilidade social e em situação de rua irão receber kit de } \\
\text { higiene e alimentação, caso se recusem ser assistidos pelos Centros de Acolhimento. }\end{array}$ \\
\hline $\mathrm{RJ}$ & $14 / 05 / 2020$ & Lei $n^{\circ} 8.826$ & $\begin{array}{l}\text { Autoriza o Poder Executivo a fornecer alimentação às pessoas abrigadas nos Centros } \\
\text { Integrados de Educação Pública (CIEPS). }\end{array}$ \\
\hline RJ & $27 / 05 / 2020$ & Lei $\mathrm{n}^{\circ} 8.848$ & $\begin{array}{l}\text { Equipes técnicas dos CRAS e CREAS devam atuar na mitigação das situações de } \\
\text { desproteção social de populações, favorecendo o acesso a alimentação. Observa-se } \\
\text { também o estabelecimento de parcerias entre secretaria de assistência social e as } \\
\text { secretarias de educação para o acesso dos usuários no recebimento de itens de alimentação } \\
\text { e higiene. }\end{array}$ \\
\hline
\end{tabular}




\begin{tabular}{|c|c|c|c|}
\hline RJ & $18 / 06 / 2020$ & Lei $\mathrm{n}^{\circ} 8.902$ & $\begin{array}{l}\text { O Poder Executivo promove campanha publicitária que estimule a doação de gêneros } \\
\text { alimentícios para organizações da sociedade civil ou entidade sem fins lucrativos que } \\
\text { desenvolvam atividades para pessoas idosas, crianças e adolescentes, pessoas com } \\
\text { deficiência e dependentes de drogas durante o período de calamidade pública. }\end{array}$ \\
\hline RJ & $08 / 07 / 2020$ & Lei $\mathrm{n}^{\circ} 8.927$ & $\begin{array}{l}\text { Estabelece ações voltadas ao acolhimento e a proteção de mulheres vítimas de violência } \\
\text { doméstica e familiar e seus dependentes, incluindo serviço de alimentação. }\end{array}$ \\
\hline RJ & $29 / 07 / 2020$ & Lei $\mathrm{n}^{\circ} 8.951$ & $\begin{array}{l}\text { Serviços essenciais estaduais que envolvem ações de solidariedade e distribuição de } \\
\text { cestas básicas de alimentos e cartôes de vale alimentação e água devem seguir todos os } \\
\text { protocolos de prevenção ao contágio da Covid-19. }\end{array}$ \\
\hline SP & $16 / 03 / 2020$ & $\begin{array}{c}\text { Decreto }^{\circ} \\
64.864\end{array}$ & $\begin{array}{l}\text { Observa a segurança alimentar dos alunos mesmo na situação de suspensão das aulas em } \\
\text { decorrência da pandemia de Covid-19. }\end{array}$ \\
\hline SP & $30 / 03 / 2020$ & $\begin{array}{c}\text { Decreto } \mathrm{n}^{\circ} \\
64.891\end{array}$ & $\begin{array}{l}\text { A rede pública estadual de ensino fornecerá alimentação aos alunos em situação de } \\
\text { pobreza. O fornecimento será efetuado a partir do pagamento de benefício ao responsável } \\
\text { legal do aluno matriculado. }\end{array}$ \\
\hline SP & $31 / 03 / 2020$ & $\begin{array}{l}\text { Decreto }^{\circ} \\
64.897\end{array}$ & $\begin{array}{l}\text { A Secretaria de Desenvolvimento Social adota providências para que sejam fornecidas } \\
\text { refeições (café da manhã, almoço e jantar) diariamente durante o período de calamidade } \\
\text { pública, através do Programa Estadual de Alimentação e Nutrição para Populações } \\
\text { Carentes. }\end{array}$ \\
\hline SP & $13 / 04 / 2020$ & $\begin{array}{c}\text { Decreto } \mathrm{n}^{\circ} \\
64.938\end{array}$ & $\begin{array}{l}\text { A Secretaria de Desenvolvimento Social realizará a ação Alimento Solidário no âmbito } \\
\text { do Projeto Família Paulista, através da distribuição de cestas básicas de alimentos às } \\
\text { famílias em situação de extrema pobreza. }\end{array}$ \\
\hline SP & $30 / 04 / 2020$ & $\begin{array}{l}\text { Lei } n^{\circ} \\
17.263\end{array}$ & $\begin{array}{l}\text { Ampliação de recursos estaduais para custear medidas que atendam a segurança } \\
\text { alimentar, e também a alimentação escolar durante o estado de calamidade pública. }\end{array}$ \\
\hline SP & $13 / 07 / 2020$ & $\begin{array}{l}\text { Lei } n^{\circ} \\
17.268\end{array}$ & $\begin{array}{l}\text { Distribuição de refeições gratuitas em unidades do Restaurante Popular para as pessoas } \\
\text { em situação de rua. O ato também relata a criação e disponibilização de linhas de crédito } \\
\text { para financiar o Fundo de Expansão do Agronegócio Paulista do Banco do Agronegócio } \\
\text { Familiar (FEAP/Benagro), como incentivo a recuperação e elevação da capacidade de } \\
\text { produção de alimentos. }\end{array}$ \\
\hline
\end{tabular}

Legenda: UF: unidade federativa; SAN: segurança alimentar e nutricional; SEJUSC: secretaria de estado de justiça, direitos humanos e cidadania; SEAS: secretaria estadual de assistência social; FPS: fundo de promoção social e erradicação da pobreza; CRAS: centro de referência de assistência social; CREAS: centro de referência especializado de assistência social.

Fonte: Autores (2021).

A partir da leitura detalhada dos atos por região (Quadro 1), nota-se que na região Nordeste as medidas foram direcionadas principalmente para o fornecimento da alimentação de qualidade aos alunos da rede pública estadual de ensino, durante o período de férias, recesso escolar e suspensão das atividades, além de ações envolvendo o fornecimento de cestas básicas às famílias socialmente vulneráveis. Verificaram-se semelhanças para algumas ações descritas nos documentos emitidos pelas regiões Norte e Nordeste, que foram as únicas a determinar limite quantitativo de bens essenciais, como a alimentação. Nessas regiões também foi observada maior atenção a auxílios financeiros e ao repasse de alimentos para populações em vulnerabilidade social. Além disso, na região Norte foi observado um ato direcionado à garantia de renda mínima aos produtores rurais.

Na região Centro-Oeste prevaleceram deliberações referentes à alimentação escolar, incluindo o repasse de alimentos cuja data de validade esteja próxima do vencimento; garantia do direito do escolar da rede pública de ensino, cadastrado e beneficiado pelo programa Bolsa Família ou equivalente, à alimentação escolar durante a suspensão das aulas em Goiás; além da articulação entre as três esferas do poder público, no estado do Mato Grosso, para assistência alimentar às famílias de estudantes da educação básica estadual ou conveniada com o Estado (Quadro 1). 
A região Sudeste, sendo a responsável pela emissão do maior número de atos, propôs ações que incluem o fornecimento de alimentos e a garantia da SAN aos alunos da rede estadual de ensino (Quadro 1). Verificou-se que um dos atos determinou o estímulo à produção e a comercialização de alimentos da agricultura familiar e de pequenos produtores, além de estimular a aquisição direta da agricultura familiar no âmbito do PNAE. Houve ainda o direcionamento de atos voltados para promover a doação de alimentos e distribuição de refeições às pessoas vulneráveis, em especial aos moradores de rua e indivíduos abrigados nos centros integrados de educação pública (Quadro 1). Esta foi a única região que publicou atos específicos para a garantia da SAN do idoso; sobre o serviço de alimentação às mulheres vítimas de violência doméstica e familiar; e sobre a garantia da SAN para as crianças e adolescentes, incluindo povos e comunidades tradicionais (Quadro 1).

Nota-se ainda que, a região Sul publicou dois atos referentes aos aspectos gerais em SAN, como a viabilização de repasses financeiros e promoção de ações intersetoriais; além de um ato que determinou a concessão de auxílio exclusivo para aquisição de gêneros alimentícios (Quadro 1).

\section{Discussão}

A capacidade de resposta liderada pelo Governo Federal para a questão da INSAN durante a pandemia de Covid-19 se mostra fragilizada, uma vez que existe uma lacuna de ações que atendam às demandas existentes (Alpino et al., 2020). A publicação da Medida Provisória n ${ }^{\circ}$ 870, de $1^{\circ}$ de janeiro de 2019 endossada pela Lei no 13.844 , de 14 de junho de 2019, culminou na extinção do CONSEA, de modo a enfraquecer o SISAN, comprometendo os processos de garantia do DHAA em todas as esferas governamentais (Castro, 2019).

A determinação por decreto, por parte do poder executivo, pode representar uma concentração do poder de decisão emergencial para lidar com a situação de INSAN. Dos 55 atos oficiais publicados, 31 destes foram emitidos na forma de decreto, indicando menor participação do poder legislativo na tomada de decisões para lidar com a emergência em decorrência da pandemia de Covid-19. Ademais, o inciso II presente no Artigo $5^{\circ}$ da Constituição Federal determina de forma precisa que “ninguém será obrigado a fazer ou deixar de fazer alguma coisa senão em virtude de lei" (Constituição da República Federativa do Brasil, 1988). Dessa forma, as ações emitidas no formato de decretos ao invés de leis, perdem o caráter notório de execução obrigatória, podendo ser interpretadas apenas como ordens ou regulamentações de execução facultativa.

A presença do termo "segurança alimentar e nutricional", descrito na íntegra ou parcialmente em 36 atos oficiais publicados, representa um avanço não apenas na incorporação desta temática na agenda governamental, mas também como um indicador da descentralização de políticas públicas atreladas à gestão pactuada. Esse processo de descentralização evidencia o papel das unidades federativas participantes do SISAN na composição de uma agenda de ações, não somente para lidar com a questão de emergência em decorrência da Covid-19, mas também um indício da pactuação federativa da PNSAN (Decreto n. 7.272, 2010).

Propostas envolvendo a articulação entre os governos estaduais e municipais foram mencionadas em 34 atos oficiais. Este achado evidencia uma ampliação do processo de descentralização das ações em SAN. Tais propostas demonstram a importância de arranjos institucionais para mobilizar diferentes setores, com vistas a garantir que as ações em SAN sejam efetivas e eficazes, enquanto durar a pandemia (Borsatto et al., 2020). Desse modo, a institucionalização do SISAN no âmbito nacional depende de mecanismos que aperfeiçoem ações intersetoriais e a descentralização da gestão pública em SAN (Vasconcellos \& Moura, 2018).

A elaboração de 37 propostas de ações abrangendo articulações intra e intersetoriais mostra-se uma estratégia para o estabelecimento de parcerias e mobilização de governos, instituições e da sociedade (Araújo \& Calazans, 2020; Malta et al., 2018). No entanto, os desmontes orçamentários e institucionais das políticas de SAN, como a extinção da Secretaria Nacional de Segurança Alimentar e Nutricional (SESAN) pelo governo federal em 2019, são fatores que dificultam a articulação de ações 
integradas, já que a SESAN tinha um papel estratégico na coordenação intersetorial da PNSAN e na descentralização do SISAN (Alpino et al., 2020; Santarelli et al., 2019). Dessa forma, instrumentos importantes que poderiam contribuir com a PNSAN, como os planos estaduais de SAN, não foram implementados, tampouco discutidos pelos governos estaduais; a exemplo do que ocorreu no estado de São Paulo, onde o CONSEA-SP se encontra vacante desde outubro de 2019 (Moreira et al., 2020).

Todas as regiões emitiram atos relacionados à alimentação escolar, possivelmente um reflexo à Lei no 13.987, de 7 de abril de 2020, que autorizou, "em caráter excepcional, durante o período de suspensão das aulas em razão de emergência ou calamidade pública, a distribuição de gêneros alimentícios adquiridos com recursos do PNAE aos pais ou responsáveis dos estudantes das escolas públicas da educação básica” (Lei n. 13.987, 2020). Essa política pública corrobora a SAN de estudantes da rede pública e consequentemente de suas famílias, uma vez que permite o repasse direto de gêneros alimentícios, através de kits de insumos para o preparo de refeições ou alimentos já preparados, que são entregues na residência com periodicidade prédeterminada. A forma de distribuição desses alimentos, assim como o recorte social priorizando famílias mais vulneráveis, ficaram a cargo da gestão local do PNAE, mantendo assim a autonomia de ações conforme a realidade de cada território (Amorim et al., 2020).

A maioria dos estados optou pelo pagamento de benefícios, para a aquisição de alimentos, diretamente aos responsáveis pelos alunos da rede pública. Todavia, não há como se ter garantia que os recursos serão utilizados para aquisição de alimentos e que estes serão de fato consumidos pelos alunos; além de que o cálculo para o repasse, baseado nos recursos do PNAE, visa suprir as necessidades de um aluno, mas provavelmente esse benefício será utilizado para toda a família (Bicalho \& Lima, 2020).

A autonomia das famílias para a escolha dos alimentos é uma realização importante do DHAA. Entretanto, são necessárias ações de Educação Alimentar e Nutricional (EAN) para evitar que tais escolhas não culminem na aquisição de alimentos nutricionalmente inadequados (Carvalho et al., 2020).

Poucos atos previram o estímulo à produção e à comercialização de insumos provenientes da agricultura familiar e de pequenos produtores, além do já previsto pelo PNAE. Essas são dimensões que, no que diz respeito à garantia da SAN, tem importante relevância para o acesso da população à uma alimentação adequada, bem como no incentivo à produção e comercialização local. Tais aspectos contribuem como uma medida protetiva contra desabastecimentos durante a pandemia, além de oferecer perspectivas de SAN a esses trabalhadores e suas famílias, que geralmente vivem em condições de vulnerabilidade social (Bocchi et al., 2019).

A distribuição de cestas básicas foi abordada em 6 atos, configurando uma das estratégias adotadas pelos governos em decorrência da suspensão das atividades ditas como não essenciais durante a pandemia. Como consequência dessas ações houve uma perda generalizada no setor econômico, gerando alta taxa de desemprego e dificultando o acesso a itens de necessidades básicas (Dias et al., 2020; Silva et al., 2020). Segundo os dados do Instituto Brasileiro de Geografia e Estatística (IBGE, 2019a), cerca de 41,3\% dos brasileiros atuavam no comércio informal no Brasil, o que é equivalente a 38 milhões de pessoas. Nesse contexto, as ações de assistência social voltadas à distribuição de cestas básicas para a população em situação de vulnerabilidade, advinda da perda de emprego e de renda ou não, torna-se um aspecto fundamental para a garantia da SAN dessas pessoas (Ribeiro-Silva et al., 2020).

A preocupação latente com o desabastecimento de alimentos aparece como influência para a emissão de 8 atos oficiais, determinando ações voltadas para o estabelecimento de limites para aquisição de produtos, incluindo alimentos. A manutenção da cadeia de fornecimento de alimentos é fundamental para a garantia da distribuição adequada desses produtos, sendo necessária a adoção de medidas emergenciais para se evitar interrupções e crises no abastecimento alimentar (Paulo Farias \& Santos Gomes, 2020).

A atual estrutura do SISAN pode, em parte, explicar a atual gestão das ações propostas pelos estados e Distrito Federal para o enfrentamento da INSAN no Brasil durante a pandemia. As execuções dos planos estaduais de SAN e da PNSAN, bem 
como o cumprimento da Lei $n^{\circ}$ 11.346, de 15 de setembro de 2006 (conhecida como Lei Orgânica de SAN - LOSAN), dependem do funcionamento do SISAN, visando assim a garantia do DHAA. Atualmente, o SISAN conta com sistema público que reúne a Câmara Interministerial de Segurança Alimentar e Nutricional (CAISAN) e suas ramificações análogas nos âmbitos distritais, estaduais e municipais, complementadas pela participação da sociedade civil através das Conferências e Conselhos de SAN (IBGE, 2019b). Em relação ao poder de atuação dos conselhos estaduais de SAN, 24 são somente de caráter consultivo (88,8\%), 13 apresentam caráter deliberativo (48,1\%) e 2 dessas entidades possuem caráter normativo $(7,4 \%)$, sendo que apenas nos estados do Amapá e Piauí estes conselhos apresentavam as funcionalidades consultivas, deliberativas, normativas e fiscalizadoras (IBGE, 2019b).

Apesar de todas as unidades federativas possuírem conselhos estaduais de SAN, os governos estaduais do Acre, Espírito Santo, Maranhão, Mato Grosso do Sul, Pará, Piauí e Santa Catarina não apresentaram propostas de ações para o combate da INSAN na pandemia de Covid-19 durante o período analisado (IBGE, 2019b; Machado et al., 2018).

Destaca-se que somente 15 unidades federativas possuem o plano estadual de SAN vigente, com o agravante de que apenas 11 estados dispunham de recursos orçamentários para viabilizar o funcionamento de suas CAISAN (IBGE, 2019b; Machado et al., 2018). A inexistência do plano estadual de SAN é um entrave importante a se considerar, uma vez que este documento auxilia os estados a comporem uma agenda de ações em SAN de acordo com as discussões realizadas na CAISAN, nos conselhos estaduais de SAN e nas Conferências de Segurança Alimentar e Nutricional (CSAN), bem como a previsão de recursos que estejam contemplados nos planos plurianuais estaduais (Machado et al., 2018).

Um dos possíveis motivos para que Minas Gerais tenha sido capaz de emitir 5 atos oficiais para ações em SAN seja o fato desse estado ser pioneiro nas criações do conselho estadual de SAN, do CAISAN estadual, e ainda na publicação do primeiro plano estadual de SAN, além de apresentar metodologia e atribuição de responsabilidades para o monitoramento deste plano (Machado et al., 2018). Ainda que Rio de Janeiro e São Paulo não possuam planos de SAN vigentes, tais estados emitiram um número expressivo de atos oficiais (7 e 6 respectivamente), estando respaldados pela existência de recursos orçamentários estaduais para os CAISAN e execução da PNSAN (Machado et al., 2018).

Cabe ressaltar que a ausência do III Plano Nacional de Segurança Alimentar e Nutricional (PLANSAN) para o período 2020-2023, cuja elaboração foi terceirizada pela contratação de consultor individual em 2019, demonstra a inoperância do governo federal para a gestão, planejamento e execução das ações intersetoriais da PNSAN (Decreto n. 7.272, 2010; Ministério da Cidadania, 2019). Desta forma, a elaboração de novos planos estaduais de SAN acaba sendo prejudicada pelo fato de não haver um documento análogo nacional como referencial de base para a implementação integrada e descentralizada das ações do SISAN.

\section{Conclusão}

Apesar das dificuldades e entraves enfrentados pelo SISAN em decorrência da falta de planejamento da agenda de SAN pelo governo federal e desmonte do CONSEA, todas as regiões do país foram capazes de elaborar propostas emergenciais para o enfrentamento da INSAN durante o período de emergência em decorrência da Covid-19. Entretanto, foi constatada uma disparidade de ações entre as unidades da federação não apenas em número, mas também na abrangência e especificidade das propostas.

A maioria dos atos oficiais foram publicados na forma de decretos, sendo entendidos como respostas emergenciais que denotam a maior concentração da tomada de decisão por parte do poder executivo, sem a devida anuência do poder legislativo estadual para mitigar a INSAN. As propostas de articulação dos governos estaduais e distrital com os municípios demonstram a intenção de ampliar e regionalizar as ações em SAN, estando em consonância com as definições da PNSAN. 
As ações baseadas em adaptações do programa de alimentação escolar e na distribuição de cestas básicas de alimentos demonstram a importância de políticas públicas permanentes em SAN, pautadas na intersetorialidade e ampla abrangência nacional.

A ausência de propostas de ações nos estados do Acre, Espírito Santo, Maranhão, Mato Grosso do Sul, Pará, Piauí e Santa Catarina no período analisado ressalta a necessidade de consolidar a incorporação da agenda de SAN, nos governos de tais entes federados, para lidar com a INSAN no contexto atual.

A emergência em decorrência da Covid-19 foi capaz de evidenciar não apenas a capacidade de resposta das unidades federativas para a questão da INSAN no Brasil, mas também os arranjos, articulações e fragilidades existentes no SISAN no âmbito estadual e distrital. Portanto, ressalta-se a importância do fortalecimento institucional e integração dos estados e Distrito Federal na gestão tripartite do SISAN, visando aumentar a capilaridade das ações em SAN e garantir o DHAA diante de situações inesperadas como a pandemia.

Os resultados e discussões tratados neste trabalho não esgotam a necessidade de novas investigações sobre os entraves na agenda governamental pactuada entre União, estados, Distrito Federal e municípios que inviabilizam a manutenção da SAN, buscando identificar os motivos para ausências, falhas e inoperâncias no âmbito do SISAN. Alinhado a tais anseios, recomendase a agregação de esforços para o monitoramento e mensuração dos efeitos da extinção do CONSEA sobre a funcionalidade do SISAN. É imprescindível averiguar os interesses e articulações dos governos para a formulação e execução de políticas públicas que contribuam para a realização do DHAA. Tais medidas se traduzem na viabilização de ações urgentes de combate à fome, $\mathrm{e}$ a outras situações e manifestações de INSAN deflagradas em momentos de crise no Brasil.

\section{Referências}

Alpino, T. M. A., Santos, C. R. B., Barros, D. C., \& Freitas, C. M. (2020). COVID-19 e (in)segurança alimentar e nutricional: Ações do Governo Federal brasileiro na pandemia frente aos desmontes orçamentários e institucionais. Cadernos de Saúde Pública, 36(8), e00161320. https://doi.org/10.1590/0102$311 \times 00161320$

Amorim, A. L. B., Ribeiro, J. R. S., \& Bandoni, D. H. (2020). Programa Nacional de Alimentação Escolar: Estratégias para enfrentar a insegurança alimentar durante e após a COVID-19. Revista de Administração Pública, 54(4), 1134-1145. https://doi.org/10.1590/0034-761220200349

Araújo, F. R., \& Calazans, D. L. M. S. (2020). Gestão das ações de segurança alimentar frente à pandemia pela COVID-19. Revista de Administração Pública, 54(4), 1123-1133. https://doi.org/10.1590/0034-761220200329

Bardin, L. (1977). Análise de conteúdo (Vol. 70). Edições 70. https://www.academia.edu/download/61102466/BARD1N_L._1977._A nalise_de_conteudo._Lisboa_edicoes_70_225.20191102-5693-11evk0e.pdf

Barreto, M. L., Barros, A. J. D., Carvalho, M. S., Codeço, C. T., Hallal, P. R. C., Medronho, R. A., Struchiner, C. J., Victora, C. G., \& Werneck, G. L. (2020). $\mathrm{O}$ que é urgente e necessário para subsidiar as políticas de enfrentamento da pandemia de COVID-19 no Brasil? Revista Brasileira de Epidemiologia, 23, e200032. https://doi.org/10.1590/1980-549720200032

Bicalho, D., \& Lima, T. M. (2020). O Programa Nacional de Alimentação Escolar como garantia do direito à alimentação no período da pandemia da COVID19. DEMETRA: Alimentação, Nutrição \& Saúde, 15, 52076. https://doi.org/10.12957/demetra.2020.52076

Bocchi, C. P., Magalhães, É. S., Rahal, L., Gentil, P., \& Gonçalves, R. S. (2019). A década da nutrição, a política de segurança alimentar e nutricional e as compras públicas da agricultura familiar no Brasil. Revista Panamericana de Salud Pública, 43, e84. https://doi.org/10.26633/RPSP.2019.84

Borsatto, R., Grigoletto, F., Macedo, A. C., \& Martensen, A. C. (2020). Respostas dos Municípios para Garantir Segurança Alimentar e Nutricional em Tempo de Pandemia. SciELO Preprints. https://doi.org/10.1590/SciELOPreprints. 163

Bowen, G. A. (2009). Document Analysis as a Qualitative Research Method. Qualitative Research Journal, 9(2), 27-40. https://doi.org/10.3316/QRJ0902027

Canesqui, A. M. (2009). Pesquisas qualitativas em nutrição e alimentação. Revista de Nutrição, 22(1), 125-139. https://doi.org/10.1590/S141552732009000100012

Carvalho, T. F. B., Sá, T. S., Ruas, J. P. P., Vieira, M. A., \& Sampaio, C. A. (2020). Nutritional status and food safety of families benefited by the bolsa familia program: Integrative review. Revista de Pesquisa Cuidado é Fundamental Online, 12, 593-602. https://doi.org/10.9789/2175-5361.rpcfo.v12.8961

Castro, I. R. R. (2019). A extinção do Conselho Nacional de Segurança Alimentar e Nutricional e a agenda de alimentação e nutrição. Cadernos de Saúde Pública, 35(2), e00009919. https://doi.org/10.1590/0102-311x00009919

Constituição da República Federativa do Brasil de 1988. (1988). http://www.planalto.gov.br/ccivil_03/constituicao/constituicao.htm 
Decreto $\mathrm{n}^{\circ}$ 7.272, de 25 de agosto de 2010. (2010). Regulamenta a Lei no 11.346, de 15 de setembro de 2006, que cria o Sistema Nacional de Segurança Alimentar e Nutricional - SISAN com vistas a assegurar o direito humano à alimentação adequada, institui a Política Nacional de Segurança Alimentar e Nutricional - PNSAN, estabelece os parâmetros para a elaboração do Plano Nacional de Segurança Alimentar e Nutricional, e dá outras providências. http://www.planalto.gov.br/ccivil_03/_ato2007-2010/2010/decreto/d7272.htm

Dias, J. A. A., Dias, M. F. S. L., Oliveira, Z. M., Freitas, L. M. A., Santos, N. C. N., \& Freitas, M. C. A. (2020). Reflexões sobre distanciamento, isolamento social e quarentena como medidas preventivas da COVID-19. Revista de Enfermagem do Centro-Oeste Mineiro, 10, e3795. https://doi.org/10.19175/recom.v10i0.3795

Erokhin, V., \& Gao, T. (2020). Impacts of COVID-19 on Trade and Economic Aspects of Food Security: Evidence from 45 Developing Countries. International Journal of Environmental Research and Public Health, 17(16), 5775. https://doi.org/10.3390/ijerph17165775

Godoy, A. S. (1995). Introdução à pesquisa qualitativa e suas possibilidades. Revista de Administração de Empresas, 35(2), 57-63. https://doi.org/10.1590/S0034-75901995000200008

Guerra, L. D. S., Cervato-Mancuso, A. M., \& Bezerra, A. C. D. (2019). Food: A disputed human right-A thematic focus for comprehension and action in food and nutritional security. Ciência \& Saúde Coletiva, 24(9), 3369. Gale Academic OneFile. https://link.gale.com/apps/doc/A603753069/AONE?sid=googleScholar\&xid=e9d4c27e

Günther, H. (2006). Pesquisa qualitativa versus pesquisa quantitativa: Esta é a questão? Psicologia: Teoria e Pesquisa, 22(2), 201-209. https://doi.org/10.1590/S0102-37722006000200010

Instituto Brasileiro de Geografia e Estatística - IBGE. (2019a). Síntese de indicadores sociais: uma análise das condições de vida da população brasileira. Coordenação de População e Indicadores Sociais. https://biblioteca.ibge.gov.br/visualizacao/livros/liv101678.pdf

Instituto Brasileiro de Geografia e Estatística - IBGE. (2019b). Perfil dos estados brasileiros: 2018. Coordenação de População e Indicadores Sociais. https://biblioteca.ibge.gov.br/index.php/biblioteca-catalogo?view=detalhes\&id=2101667

Jaime, P. C. (2020). Pandemia de COVID19: Implicações para (in)segurança alimentar e nutricional. Ciência \& Saúde Coletiva, $25(7)$, 2505. https://doi.org/10.1590/1413-81232020257.12852020

Kripka, R. M. L., Scheller, M., \& Bonotto, D. L. (2015a). Pesquisa Documental: Considerações sobre conceitos e características na Pesquisa Qualitativa. CIAIQ2015, 2. https://proceedings.ciaiq.org/index.php/ciaiq2015/article/view/252

Kripka, R. M. L., Scheller, M., \& Bonotto, D. L. (2015b). Pesquisa documental na pesquisa qualitativa: Conceitos e caracterização. Revista de Investigaciones UNAD, 14(2), 55. https://doi.org/10.22490/25391887.1455

Lei $\mathrm{n}^{\circ}$ 11.346, de 15 de setembro de 2006. (2006). Cria o Sistema Nacional de Segurança Alimentar e Nutricional - SISAN com vistas a assegurar o direito humano à alimentação adequada e dá outras providências. http://www.planalto.gov.br/ccivil_03/_ato2004-2006/2006/lei/111346.htm

Lei $\mathrm{n}^{\circ}$ 13.987, de 07 de abril de 2020. (2020). Altera a Lei $\mathrm{n}^{\circ}$ 11.947, de 16 de junho de 2009, para autorizar, em caráter excepcional, durante o período de suspensão das aulas em razão de situação de emergência ou calamidade pública, a distribuição de gêneros alimentícios adquiridos com recursos do Programa Nacional de Alimentação Escolar (PNAE) aos pais ou responsáveis dos estudantes das escolas públicas de educação básica. http://www.planalto.gov.br/ccivil_03/_ato2019-2022/2020/lei/113987.htm

Machado, M. L., Gabriel, C. G., Soar, C., Mamed, G. R., Machado, P. M. O., Lacerda, J. T., Martins, M. C., \& Marcon, M. C. (2018). Adequação normativa dos planos estaduais de segurança alimentar e nutricional no Brasil. Cadernos de Saúde Pública, 34(1), e00206716. https://doi.org/10.1590/0102-311x00206716

Malta, D. C., Reis, A. A. C., Jaime, P. C., Morais Neto, O. L., Silva, M. M. A., \& Akerman, M. (2018). O SUS e a Política Nacional de Promoção da Saúde: Perspectiva resultados, avanços e desafios em tempos de crise. Ciência \& Saúde Coletiva, 23(6), 1799-1809. https://doi.org/10.1590/141381232018236.04782018

Ministério da Cidadania. (2016). Transformando nosso mundo: a agenda 2030 para o desenvolvimento sustentável. http://www.mds.gov.br/webarquivos/publicacao/brasil_amigo_pesso_idosa/agenda2030.pdf

Ministério da Cidadania. (2019). Contratação de consultor individual modalidade produto - IICA BRA/IICA/17/001. Comissão Técnica Portaria No 78/SEISP/SP. http://www.mds.gov.br/webarquivos/cidadania/editais/organismos_internacionais/IICA/Site_edital\%20143_IICA.pdf

Ministério do Planejamento, Desenvolvimento e Gestão. (2018). Agenda 2030: ODS-Metas nacionais dos objetivos de desenvolvimento sustentável. Instituto de Pesquisa Econômica Aplicada. http://repositorio.ipea.gov.br/bitstream/11058/8855/1/Agenda_2030_ods_metas_nac_do s_obj_de_desenv_susten_propos_de_adequa.pdf

Moreira, R. M., Nunes, K. R., Baccarin, J. G., \& Stamato, B. (2020). Políticas públicas do estado de São Paulo e a sociedade civil organizada no enfrentamento dos impactos da COVID-19 sobre os sistemas alimentares: O caso do PLANSAN-SP. Simbio-Logias Revista Eletrônica de Educação Filosofia e Nutrição, 12(16), 158-183. https://doi.org/10.32905/19833253.2020.12.16p158

Paulo Farias, D., \& Santos Gomes, M. G. (2020). COVID-19 outbreak: What should be done to avoid food shortages? Trends in Food Science \& Technology, 102, 291-292. https://doi.org/10.1016/j.tifs.2020.06.007

Ribeiro-Silva, R. C., Pereira, M., Campello, T., Aragão, É., Guimarães, J. M. M., Ferreira, A. J., Barreto, M. L., \& Santos, S. M. C. (2020). Implicações da pandemia COVID-19 para a segurança alimentar e nutricional no Brasil. Ciência \& Saúde Coletiva, 25(9), 3421-3430. https://doi.org/10.1590/141381232020259.22152020

Santarelli M., David G., Burity V., \& Rocha, N. C. (2019). Informe Dhana 2019: autoritarismo, negação de direitos e fome. Brasília: FIAN Brasil 2019. https://fianbrasil.org.br/wp-content/uploads/2019/09/Resumo-Executivo-DHANA-PORTUG\%C3\%8AS.pdf 
Research, Society and Development, v. 10, n. 9, e7910917827, 2021

(CC BY 4.0) | ISSN 2525-3409 | DOI: http://dx.doi.org/10.33448/rsd-v10i8.17827

Silva Filho, O. J., \& Gomes Júnior, N. N. (2020). O amanhã vai à mesa: Abastecimento alimentar e COVID-19. Cadernos de Saúde Pública, 36(5), e00095220. https://doi.org/10.1590/0102-311x00095220

Silva, L. L. S., Lima, A. F. R., Polli, D. A., Razia, P. F. S., Pavão, L. F. A., Cavalcanti, M. A. F. H., \& Toscano, C. M. (2020). Medidas de distanciamento social para o enfrentamento da COVID-19 no Brasil: Caracterização e análise epidemiológica por estado. Cadernos de Saúde Pública, 36(9), e00185020. https://doi.org/10.1590/0102-311x00185020

Sipioni, M. E., Riquieri, M. R. L., Barbosa, J. P. M., Biscotto, D. B., Sarti, T. D., \& Andrade, M. A. C. (2020). Máscaras cobrem o rosto, a fome desmascara o resto: Covid-19 e o enfrentamento à fome no Brasil. Preprints. https://doi.org/10.1590/SciELOPreprints.660

Vasconcellos, A. B. P. A., \& Moura, L. B. A. (2018). Segurança alimentar e nutricional: Uma análise da situação da descentralização de sua política pública nacional. Cadernos de Saúde Pública, 34(2), e00206816. https://doi.org/10.1590/0102-311x00206816 